\title{
The silent pandemic
}

Addressing antimicrobial resistance

Antimicrobial resistance (AMR) is a silent pandemic that poses a huge threat to global health and development. The World Health Organization and its

partner organisations are
seeking to raise awareness of th seeking to raise awareness of the
issue. One approach is through issue. One approach is through
annual monitoring of progress annual monitoring of progress
through the Tripartite AMR Country Self-Assessment Survey
(TrACSS), for which the 2021 results are now available, www. amrcountryprogress.org. While significant progress has
been made, the COVID-19 been made, the COVID-19 pandemic has weakened countries' ability to address AMR. The data highlights the that lack effective and functional multisectoral working groups, monitoring and evaluation frameworks, infection control measures, awareness campaigns, or comprehensive training for health workers. Ultimately, this requires sustained political and

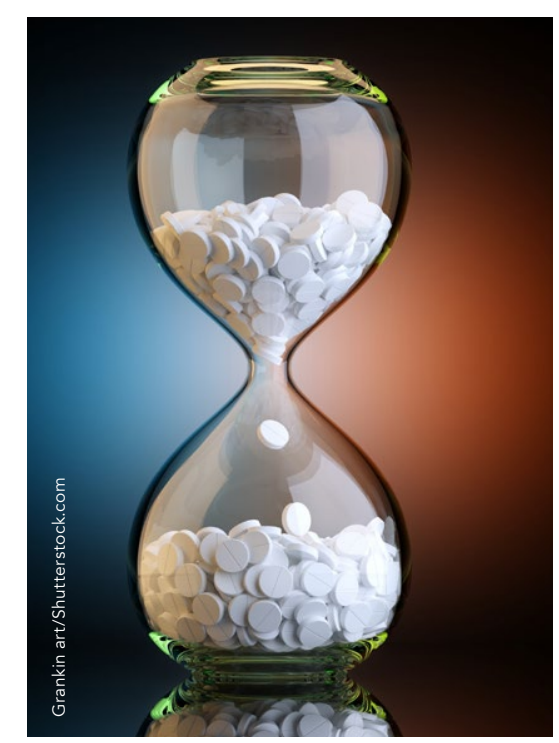
or almost two years, time has
seemingly stood still, with the
COVID-19 pandemic raging around us. In an era of unparalleled connectedness, mankind has been simultaneously pulled together and thrown apart by the enormity of the impacts. However, COVID-19 is not the only major health threat that we face, fighting Antimicrobia resistane, AMR, is a silent pandemic known to and understood by only a small number of people, its effects on humankind are profound.

To coincide with World Antimicrobial Awareness Week (WAAW), held annually in November, the World Healt Organization (WHO) and its partner organisations, the Food and Agriculture Organization of the United Nations (FAO), and the World Organisation for Animal Health (OIE), collectively called the Tripartite, are seeking to raise awareness of the issue, and to highlight the progress

\section{MIRACLE DRUGS FACING}

\section{GROWING RESISTANCE}

Hailed as miracle drugs, antimicrobials

the 20th century Natural products with

antimicrobial activity (such as herbs

and honey) have been recognised for
millennia. However, it was not until the

'discovery' of bacteria (by Antonie Philips van Leeuwenhoek) in the 17th century, and recognition that bacteria are related to disease (via the work of Robert Koch and Louis Pasteur) in the late 1800s, tha a more scientific approach was taken. The first really powerful antimicrobia drug discovered was the antibiotic penicilin, a substance that fundamentally changed medicine. First discovered by widely available following, tit becam widely available following the adven mid-1940s. Since then, countless other antimicrobials, including antibiotics, antivirals, antifungals, and antiparasitics, have been developed.

However, today, less than a century later humans are becoming the victims of our own success. A nising tide of AMR, which occurs when bacteria, viruses, fungi, and parasites naturally evolve to no longer ability to treat infections.

The main driver of AMR is the misuse and overuse of antimicrobials; however, poor sanitation, poor infection prevention and control practices, and lack of access to clean water, have exacerbated the issue by facilitating the spread of treatmentresistant microbes. High rates of drugresistance are now observed for many common bacterial infections such as tuberculosis, urinary tract infections, and pneumonia. For example, in many parts of the world, fluoroquinolones (a class of antibiotics) to treat E. colia are now ore than half of patients.

\section{GLOBAL ACTION PLAN ON}

\section{ANTIMICROBIAL RESISTANCE}

In the call to action against AMR, the

resistance (GAP-AMR) with five strategic

objectives, was adopted by all countries in 2015. To track progress of the GAPAMR, the Tripartite AMR Country SeltAssessment Survey (TrACSS) is focused on monitoring and evaluating the implementation of national action plans (NAPs). Currently on its fifth iteration, the TrACSS survey is completed annually, with each country asked to submit an official response.

\section{MAKING TrACSS}

The survey starts by assessing the multisectoral working goning of and national antimicrobial regulations. It then addresses four of the five GAP-AMR strategic objectives: 1) raising awareness; sureillance and research; 3) roducing infections: and 4) optimising antimicrobial medicine use.

2021 TrACSS SUMMARY HUMAN HEALTH

The results of the fifth (2021) TrACSS survey have recently been made available (www.amrcountryprogress.org), with a record number of 163 countries completing the survey (accounting for $84 \%$ of the total 194 WHO member states). It is noteworthy that all countries ofWHO's Eastern Mediterranean and South East Asia regions submitted a esponse, almost all countries from the European region (94\%) responded, and a from the African region $(87 \%)$, the Americas (66\%) and the Wester Pacif Region (63\%) compared to last year. Link Region $(63 \%)$ comp
to summary slides.

Effective multisectoral governance is essential for implementing NAPs. In total, 82 countries have established functional multisectoral working groups. However, 68 countries report that while multisectoral working groups have been established, they are not yet functiona. Of the responding countries in 202 there have been greater advances in terms of NAPs, with 140 countries reporting a developed NAP, 95 countries implementing that NAP, and 32 actively monitoning heir mplementation. Notably more than $90 \%$ of the global population NAPs on AMR, with all countries reporting that the human health sector is actively involved in the development and

implementation of NAPs. However only

46 countries have NAPs that involve all relevant sectors (ie, human health, anima health, food safety, environment, food production, and plant health).

Addressing AMR within the human health sector requires the involvement of various mitiatives. More than $60 \%$ of countries have NAPs linked to other health topics, with the most common being food safety and security ( $n=66,40 \%$ of countries), , (n) $(n=54,33 \%$ of councics), and health lesser linked topics ( $n=15-45,9-28 \%$ of

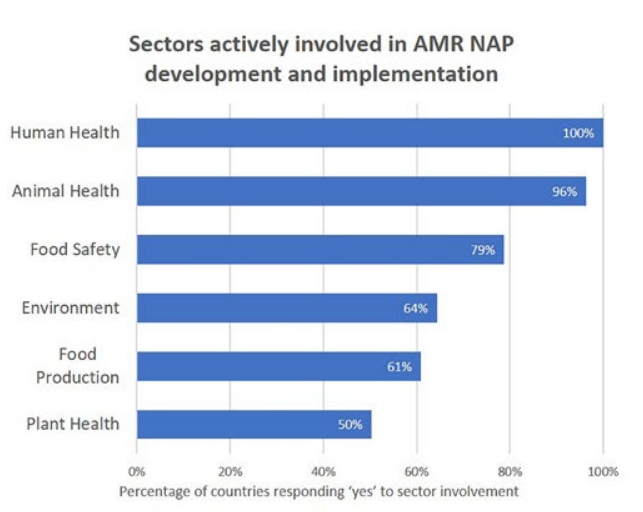

Are AMR NAPs linked to action plans
of other health topics?

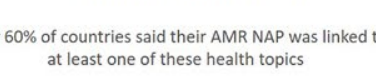

AMR National Action Plans: linkages to sectors and other health topics

countries) include water, sanitation and hygiene (WASH), sexually transmitted infections, immunisation, malaria, national environmental plans, and neglected tropical diseases.

GAP objective 1 - Raising awareness. Of responding countries in 2021,65 countries (39\%) report governm supported nationwide AMR awareness campaigns targeting all key (47\%) report having more limited, small-scale AMR awareness campaigns targeting some relevant stakeholders, mainly in the human health sector. In terms of training on AMR, 128 countries (79\%) report that AMR is covered in at least some training for human health

the indicators within TrACSS, monitoring for antimicrobial sales and consumption has the highest number of countries (n=38, or $23 \%$ ) at Level $\mathrm{A}$, that is, no established capacity for monitoring

There is progress in surveillance systems of antimicrobial resistance in huma $(n=118,73 \%)$ are collating AMR data at least nationally, with over half of responding countries $(n=87,54 \%)$ having a standardised national AMR surveillance system for data collection and reporting. A total of 18 countries (11\%) report being at the highest level, having an AMR surveillance system that

In the call to action against antimicrobial resistance, one weapon is TrACSS -

the Tripartite AMR country Self-Assessment Survey.

workers; however, less than $10 \%$, or 17 countries, have AMR systematically incorporated into their curricula.

GAP Objective 2Monitoring and surveillance. Despite the majority of countries having
laws and regulations governing the prescription and sale of antibiotics $(91 \%$, or 148 countries with regard to human health), the ability to monitor these regulations remains a challenge, highlighting a clear discrepancy betwe the situation on paper and the reality on the ground. Of 148 countries with heath, just 89 an ations for human antimicrobial sales ata montor consumption and/or use data for human strong laboratory network, but just 52 countries (33\%) report a national AMR laboratory network in human health at an advanced capacity Level E (ie, a Nationa Reference Laboratory that supports a bacteriology laboratory network for the identification of pathogens and AMR trough a systematic approach to cascade including a National External Quality Assurance programme).

GAP objective 3-Reducing AMR infections in human health. the 
infection prevention and control (IPC) programmes. However, most have in program facities, and 33\% of countries ramin that is, no national IPC programme, or one exists but is not disseminated or implemented). The data show that $28 \%$ and $26 \%$ of countries have NAPs that are linked to WASH and to immunisation, respectively.

GAP objective 4-

Optimising antimicrobial use.

$72 \%$ of countries $(n=115)$ are actively implementing policies and practices for optimising antimicrobial use in at least some healthcare facilities with guidelines for appropriate antimicrobia use available; and only around $6 \%$ of systemically feeding Levol (hat is, prescribers). A powerful tool to promote optimising antimicrobial use is WHO's AWaRe classification, which classifies antibiotics into three groups to guide optimal use and monitoring. In the first group (Access), antibiotics are available as a first or second choice for treatment; in the second group (Watch) antibiotics are recommended for only specific

A rising threatens use owing to potential drug-resistan finally, in the third group (Reserve), antibiotics should only be used to treat infections caused by multidrug-resiste organisms. Most antibiotics listed as fall into the Watch for human medicine fall into the Watch group. As of 2021, the AWaRe classification in their

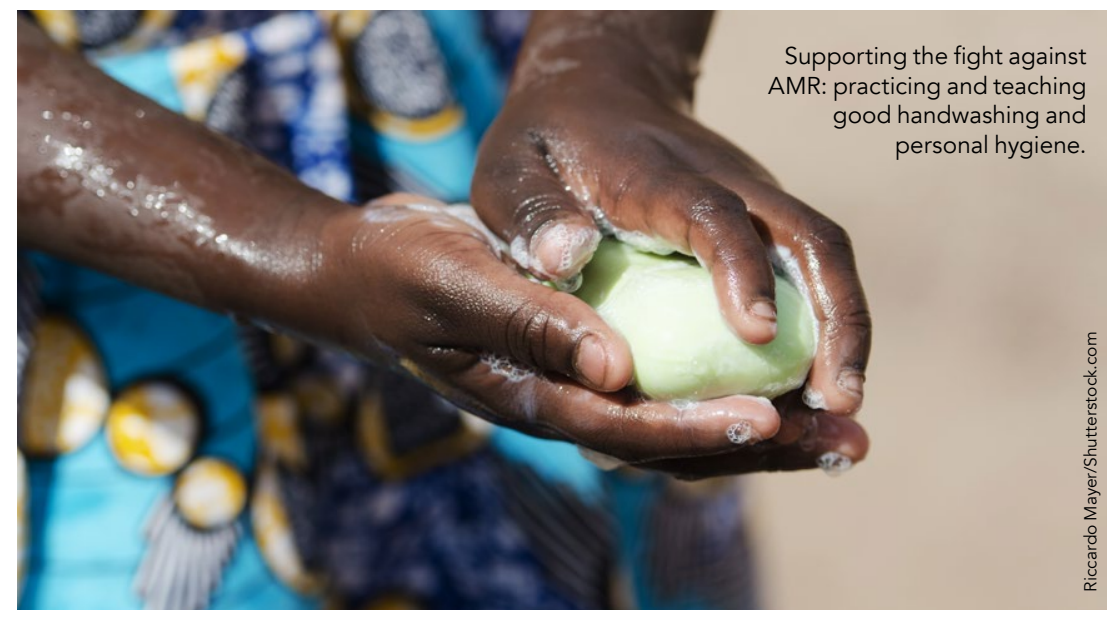

national essential medicines list (EML). Worryingly, 17 countries are in Level $A$ having no knowled

\section{IMPACT OF COVID-19}

As with most facets of life, COVID-19 fourth TrACSS survey in 2020 saw an $11.8 \%$ decrease in response rate. Thankfully, the number of countries engaging with the TrACSS survey rebounded from 136 in 2020 to

impacts (eg, deferral of data collection, MOVING FORWARD Addressing AMR requires a
multitude of targeted actions that are interdependent. So the clear take-home message from the 2021 TrACSS survey is the need to focus on providing support to countries through a programmatic approach to ensure effective implementation of NAPs. Based on the large gaps identified through the survey targeted efforts are
also needed for $42 \%$ $=68$ ) of countries with multisectoral working groups that 163 in 2021. However, 151 of those are not yet functional, the $45 \%(n=74)$ countries (93\%) reported that the of countries with no plan or with an COVID-19 pandemic and response unimplemented plan for monitoring had impacted on NAP development antimicrobial sales, and the $33 \%(n=54)$ and implementation. Among them, of countries with no IPC plan or with on $134(82 \%)$ cited governance and that is not yet implemented. Data also funding deferred committee meetings, nuggests that more technical support is etc), and 128 (79\%) cited operational their AMR diagnosis and bacteriology laboratory networks this is essential to generate evidence to reinforce IPC measures in healthcare facilities, adapt antimicrobial stewardship policies, and other areas of NAP implementation. Countries could also benefit from technical support for developing targeted awareness campaigns, providing training (in particular, systematic inclusion of AMR in health workforce curricula), and integrating AMR activities into other national health and development strategies, including to achieve the sustainable development goals. However, ultimately, these actions and adequate financing.

\section{Behind the Research \% \\ Pravarsha \\ Prakash

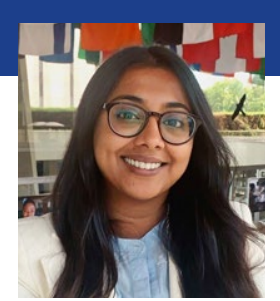 \\ Ponnu \\ Padiyara}

Research Objectives

To improve awareness and understanding of the global implementation of national action plans for addressing antimicrobial resistance in human health.

\section{Detail}

AMR Division, Surveillance, Prevention and 20 Avenue Appia, 1211 Geneva Switzerland

Bio

Pravarsha Prakash is a Technical Officer fimicrobial resistance (An) Division the World Health Organization. With strong interests in the One-Health aspects of
AMR, she manages all aspects of the globa Tripartite AMR Country Self-Assessment Survey, which is jointly administered by WHO annually along with the Food and Nations and World Organisation for Animal The publish the global Tripartite AMR suppommendedindion frame amp and support to countries to establish their monitoring systems for their multisectora

Pravarsha holds a Master of International Healthcare Management, Economics and an MSc in Microbiology from Saint Joseph's College, Bangalore, India.

Ponnu Padiyara is a Technical Officer for monitoring and evaluation in the att the World Health Organ Division manages the analysis and reporting of the results of the Tripartite AMR Country collection of data and analysis of various global AMR indicators, and supports monitoring and
action plans.

Ponnu holds an MPH from Yale University and is currently completing her DrPH in Health Security from Johns Hopkins intersection between AMR and gin the

Collaborators
Food and Agriculture Organization of the World Organisation for Animal Healt

\section{References}

-WHO Webinar: Tripartite AMR Country Self-Assessment Survey (TrACSS) Results 2021. www.who.int/teams/ series - Davey,

news, medicar of Antimicrobial Discovery. www. Discovery.aspx World Health Orga www.who.int/news-room/fact-sheets/detail// antimicrobial-resistance

-World Health Organization. World Antimicrobial Awareness Week: 18-24 November 2021. www. who.int/campaigns/world-antimicrobial-awarenessweek/2021

World Health Organization (2020) Monitoring global progress on antimicrobial resistance: Tripartite AMR Country Self-Assessment Survey (TrACSS) 2019-2020 Global analysis report. WHO Press, Geneva - World Health Organization AMR Division (2021) Results (TrACSS) 2021. Power Point presentation Sunve

\section{Personal Response}

As individuals, what can we do to support the fight Ast AMR?

II An important way to protect against drug-resistant - practicing and teaching good handwashing and personal hygiene

- preparing food safely

mising close contact when you or others are sick to date

- practicing safer sex. If you do get sick, you can also
antimicrobial resistance byy

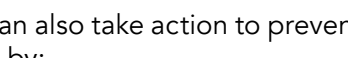
- Only using antimicrobials when prescribed by a health
worker and never using or sharing leftover antimicrobials with others.

Wecome an AMR champio 列 - participate in local, national, and global awareness and Call- 